\title{
QBism and the limits of scientific realism
}

\author{
David Glick ${ }^{1,2}$ (ID
}

Received: 17 March 2020 / Accepted: 26 March 2021 / Published online: 24 May 2021

(C) The Author(s) 2021

\begin{abstract}
QBism is an agent-centered interpretation of quantum theory. It rejects the notion that quantum theory provides a God's eye description of reality and claims instead that it imposes constraints on agents' subjective degrees of belief. QBism's emphasis on subjective belief has led critics to dismiss it as antirealism or instrumentalism, or even, idealism or solipsism. The aim of this paper is to consider the relation of QBism to scientific realism. I argue that while QBism is an unhappy fit with a standard way of thinking about scientific realism, an alternative conception I call "perspectival normative realism" may allow for a reconciliation.
\end{abstract}

Keywords QBism · Quantum Bayesianism · Realism · Perspectivalism · Normativity

\section{Introduction}

QBism is an interpretation inspired by the central role played by information in contemporary applications of quantum theory. Accordingly, the emphasis of QBists is on the application of quantum theory by agents intervening on the world. The starting idea of QBism—which originally stood for "quantum Bayesianism" — is that the probabilities delivered by quantum theory are to be understood as degrees of belief along the lines of the subjective Bayesian approach to probability (Caves et al. 2002). Since its original proposal, the view has evolved and attracted new followers in the physics community, but has been less warmly received by philosophers. Many, it seems, share the view of Hagar (2003) that "Fuchs' 'thin' realism, and the entire

David Glick

daglick@ucdavis.edu

1 Stellenbosch Institute for Advanced Study (STIAS), Wallenberg Research Centre at Stellenbosch University, Stellenbosch, South Africa

2 Department of Philosophy, University of California, Davis, CA, USA 
'fog from the north' which inspires it, are nothing but instrumentalism in disguise" (p.772). ${ }^{1}$ However, this is at odds with the avowed realism of QBists such as Fuchs (2017b) and Mermin (2014).

The aim of this paper is to clarify the status of QBism with respect to scientific realism. I argue that QBism's claim to realism can be justified by understanding the view as a form of perspectival normative realism. This issue is of importance not just for those with an interest in QBism and other "non-ontic" approaches to quantum theory (e.g., Boge 2018; Friederich 2013; 2015; Healey 2012; 2017b), but also serves to test the limits of scientific realism in the context of quantum theory. QBism proposes a non-standard understanding of the relation of a scientific theory to the world, and as such, casts the question of realism in a new light. At the very least, the following investigation should make clear that the question of realism about quantum theory is more complex than it may at first seem. ${ }^{2}$

The paper proceeds as follows. Section 2 introduces QBism and some of its motivations. Next, Section 3 considers whether QBism can be viewed as a form of standard scientific realism. The upshot of that discussion is that QBism, while not inconsistent with it, is an unhappy fit with this conception of scientific realism. In particular, standard scientific realism fails to adequately account for the perspectival and normative aspects of QBism. These aspects of the view are discussed in Sections 4 and 5, respectively. Taking these aspects seriously leads to a novel conception of scientific realism: perspectival normative realism, which provides the basis for a better understanding of QBism. Section 6 concludes by arguing that, so understood, QBism is able to meet the realist demands appropriate for quantum theory.

\section{What is QBism?}

The empirical support for quantum theory consists in the success of its probabilistic predictions. Every opportunity taken to test quantum theory has vindicated its predictions over those of potential rivals. In the standard formalism, these predictions follow from the quantum state ascribed to a system and the Born rule. QBism maintains that these probabilities are subjective degrees of belief of some agent concerning what she will experience, e.g., upon performing a measurement of some observable. Moreover, the sole function of the quantum state is to encode the agent's information

\footnotetext{
${ }^{1}$ Compared to other interpretations (e.g., Everett, de Broglie-Bohm, GRW), QBism has received considerably less attention from philosophers. Among those who have engaged with QBism, most have done so with the aim of highlighting problems with the view (Bacciagaluppi 2014; Brown 2019; Earman 2019; Norsen 2016). Even (Timpson 2008), who defends QBism from the charges of idealism and solipsism, also points to its explanatory shortcomings. Note that these authors do not necessarily agree with Hagar's "instrumentalism in disguise" claim, but it remains commonplace among philosophers to take QBism to be opposed to scientific realism but it remains commonplace among philosophers to take QBism to be opposed to scientific realism about quantum theory. For example, in his Stanford Encyclopedia entry (Myrvold 2018) categorizes QBism as a "non-realist approach to quantum mechanics."

${ }^{2}$ Cf., Healey (2020), who attempts to broaden the understanding of scientific realism along pragmatist lines to accommodate his account of quantum theory.
} 
(or subjective beliefs) associated with the system. ${ }^{3}$ So why would one adopt this kind of subjectivist interpretation of quantum theory?

There are several kinds of arguments put forth by QBists. One family of arguments notes the unifying power of taking the quantum state to be epistemic. The idea is that certain puzzling features of quantum theory follow naturally from an epistemic approach. A notable example here is Spekkens (2007), which seeks to show that a purely classical theory-Spekkens's toy model-can reproduce a number of "quantum" effects if states are understood epistemically. For example, the no cloning theorem that prohibits perfect duplication of a quantum state is shown to hold in the toy model. Of course, Spekkens's model is quite limited in its application and cannot recover the full content of quantum theory. Nevertheless, QBists maintain that it goes far enough to demonstrate the explanatory virtues of an epistemic view of quantum states; features such as no-cloning that appear unexplained or ad hoc on the ontic view are given a natural explanation on the epistemic view. ${ }^{4}$

The other sort of argument maintains that various conceptual puzzles that dog the interpretation of quantum theory are dissolved by adopting the subjective epistemic viewpoint. According to advocates, the process of measurement and the presence of non-local phenomena are described simply and without paradox by QBism. Measurement involves simply updating one's subjective beliefs about future experiences on the basis of new information. Non-locality doesn't arise in an EPR experiment because each agent-Alice, Bob, an agent at the source-has their own quantum state which reflects their individual beliefs. A distant measurement has no effect on an agent's beliefs (until they become aware of it), so nothing in the vicinity of Bob changes when Alice performs her measurement. 5

QBism also manages to avoid the various "no go" results surrounding epistemic approaches. For instance, the PBR theorem (Pusey et al. 2012) rules out a version of the epistemic approach in which the quantum state represents our uncertainty about

\footnotetext{
${ }^{3}$ The QBist should avoid saying that the quantum state encodes beliefs about the system because this suggests that the system has an underlying ontic state that is the target of the agent's beliefs. As discussed below, this “ $\Psi$-epistemic ontological model"(Harrigan and Spekkens 2010) runs into difficulties, such as the no go theorem of Pusey et al. (2012). So, strictly speaking, the quantum state encodes an agent's beliefs about their future experiences that are associated with the physical system-e.g., what they will experience when performing a measurement of it. That said, it will often be useful to speak of "measurement outcomes" and "the quantum state of a system," which should be taken to be elliptical for more careful expressions of the sort just mentioned.

${ }^{4}$ It's not entirely clear that QBists can appeal to the explanatory power of Spekkens's toy model given that the latter presupposes the ontological models framework that QBists reject. Fuchs indicates that Spekkens's toy model provides a general sense in which "far from being an appendage cheaply tacked on to the theory, the idea of quantum states as information has a simple unifying power that goes some way toward explaining why the theory has the very mathematical structure it does" (Fuchs 2010, p.3). However, much of that unifying power seems to depend on the " $\Psi$-epistemic ontological model" conception of quantum states mentioned in the previous footnote. Thanks to an anonymous referee for raising this worry. ${ }^{5}$ This does not preclude the presence of some form of non-locality. For instance, one may argue that when Alice and Bob compare results, they will find non-local correlations in measurement outcomes. The QBist may wish to treat such a revelation as simply Alice performing another (local) measurement, but doing so relegates Bob's testimony to merely an aspect of Alice's experience, arguably moving us closer to solipsism (see, Norsen 2016; Earman 2019).
} 
the underlying ontic state of a physical system. QBism avoids the PBR theorem by rejecting this understanding of epistemic states. QBism rejects that there is an underlying ontic state about which we are unsure, rather, it contends that we have degrees of belief about our own future experiences only. This may lead to charges of idealism or solipsism, but the QBist need only deny that quantum states encode credences over unknown ontic states of the physical systems to which they are applied. The QBist is free to believe that objective reality exists and that quantum theory can tell us about it in some other, more indirect manner.

In sum, QBism offers an account of quantum theory in which agents use the theory to guide their degrees of belief about potential future experiences. QBists reject the standard realist assumption that to interpret quantum theory is to say how it describes external reality. Indeed, QBists often forsake the external, "God's eye” perspective altogether in favor of a diversity of different agent-centered perspectives on the quantum world.

\section{QBism as standard scientific realism?}

While there remains disagreement about the correct formulation of scientific realism, many follow Psillos (1999) in identifying three main components of the view, roughly:

Metaphysical realism: a recognition of the existence of a mind-independent reality;

Semantic realism: a commitment to interpret theories literally;

Epistemic claim: a commitment to the approximate truth of our best confirmed theories.

As noted above, QBism is occasionally dismissed as solipsism or idealism. After all, on this view quantum theory functions as a user's manual that guides an agent's beliefs about their own future experiences. However, the QBist needn't deny that there is a reality external to the agent. Indeed, Fuchs offers the following support for metaphysical realism:

We believe in a world external to ourselves precisely because we find ourselves getting unpredictable kicks (from the world) all the time. (Fuchs 2017b)

What QBism denies is that models in quantum theory should be viewed as third-personal descriptions of external reality, but this doesn't bear on metaphysical realism, which they are free to adopt for other reasons. Assessing the status of the other two aspects of standard scientific realism with respect to QBism is less straightforward. First, consider semantic realism. The requirement to take a theory literally fits most naturally with scientific theories presented in linguistic form, where semantic realism is intended to rule out views such as logical positivism that seek to reinterpret certain sentences so as to avoid making reference to putative unobservable entities. It's unclear, however, how to apply this idea to the formalism and 
mathematical models that are the target of quantum interpretations. ${ }^{6}$ For instance, Wallace (2012, p.13) claims that taking quantum theory literally yields a multiverse of quasi-classical worlds, but others reject this characterization. Indeed, QBists may argue that their view is the result of "accepting the Bell/EPR analysis at face value" (270 ; Caves et al. 2007) or taking literally what founders like Bohr said about the theory (Fuchs 2017a). ${ }^{7}$ These examples demonstrate the ambiguity involved in the concept of a "literal construal" of quantum theory.

Second, the concept of approximate truth that figures in the epistemic claim also requires explication. In the context of a linguistically-presented scientific theory, perhaps this can be made out in terms of the successful reference of central terms (Laudan 1981), but on a model-based approach the notion is harder to pin down. One must locate some relation between models and the world capable of playing an analogous role to successful reference in the linguistic case.

A popular approach is to adopt a form of selective scientific realism, which maintains that our best theories can tell us about the world, but is careful to distinguish elements of theoretical models that are genuine reflections of reality from those that are merely artifacts of the representation. This task requires substantial interpretative work and its results are often controversial, but so long as one preserves the sense that models have latched onto the structure of the world, one preserves the standard conception of scientific realism: there is an external reality and our theories, taken literally, accurately describe it (more or less).

There are several varieties of selective scientific realism. Structural realism, for instance, maintains that models capture only the structure of the world. Thus, individual elements of theoretical models needn't correspond to individual entities, but structural or relational features do have worldly counterparts. For example, Worrall (1989) claims that Fresnel's equations do not describe genuine physical properties of a luminiferous ether-as Fresnel supposed-but rather, reflect the structure underlying optical phenomena. Other versions of this approach of selective skepticism are possible as well. One might countenance only entities with certain causal features (Hacking 1983), or those involved in novel predictive successes (Psillos 1999). Any such view of differential ontological commitment counts as selective scientific realism as understood here. ${ }^{8}$

\footnotetext{
${ }^{6}$ On van Fraassen's (1980) presentation of the semantic view, there is a clear sense in which models may be taken literally, but this is because the models in question already presuppose a specific physical interpretation. In the case of quantum theory, the models van Fraassen has in mind are ways the world could be that satisfy the quantum axioms (1980, p.65). However, this notion of models isn't applicable to the requirement of semantic realism as it applies to an interpretation of quantum theory like QBism. Here what is needed is a notion of literal construal applicable to the models that form the common target of quantum interpretation — say, density operators on Hilbert space-not the models that result from such an interpretation. For a recent discussion of van Fraassen's understanding of quantum interpretation that touches on literalism, see Wolff (2020).

${ }^{7}$ Thanks to an anonymous referee for this suggestion.

${ }^{8}$ One might add a further requirement that the extent of ontological commitment must reach some threshold, below which models are simply too remote from reality to qualify as faithful representations. I discuss the implications of this for QBism below.
} 
Is QBism compatible with selective scientific realism? Initially, one may think not. After all, QBists deny that core elements of quantum models-quantum states and their evolution-correspond to elements of reality. However, Fuchs urges that

...there is more to quantum mechanics than just three isolated terms (states, evolution, and measurement)- there's the full-blown theory that glues these notions together in a very particular way, and in a way that would have never been discovered without empirical science (Fuchs 2017b).

More specifically, recently QBists have placed increasing emphasis on the place of the Born rule as an objective feature of reality. The Born rule is typically formulated as a function that takes a quantum state to the probability of an observable taking a certain value or the occurrence of a certain measurement outcome. For example, one may write the Born rule for finding an eigenvalue $k_{i}$ of an observable $O$ for a system in a pure state $\psi$ :

$$
p\left(k_{i} \mid \psi\right)=\left\langle\psi\left|P_{i}\right| \psi\right\rangle=\left|\left\langle k_{i} \mid \psi\right\rangle\right|^{2}
$$

where $P_{i}$ is the projection onto the eigenspace of $O$ corresponding to $k_{i}$. However, given their focus on subjective probabilities, QBists have sought to eliminate reference to the misleading quantum state and formulate the Born rule entirely in terms of probabilities, understood as subjective degrees of belief about (experiences of) measurement outcomes. The resulting version of the Born rule is what QBists now call the "Essential Representation" (DeBrota et al. 2020).

The Essential Representation is cast in terms of hypothetical measurements called SICs (Fuchs 2010; Fuchs and Schack 2013). Before describing SICs, we should note that the Born rule can be stated in terms of more general measurements than those associated with a projection onto the eigenspace of some Hermitian operator as assumed in Eq. 1. A positive operator-valued measure (POVM) is a collection of positive semi-definite operators $E_{i}$ on a Hilbert space $H$ such that $\sum_{i} E_{i}=I$. In terms of POVMs, the Born rule takes the simple form:

$$
p(i \mid \rho)=\operatorname{tr} \rho E_{i}
$$

for an outcome $i$ and an arbitrary quantum state $\rho$. A SIC is a symmetric, informationally complete POVM. Formally, a SIC is a set of $d^{2}$ rank-one projection operators $\Pi_{i}=\left|\psi_{i}\right\rangle\left\langle\psi_{i}\right|$ on a finite $d$-dimensional Hilbert space such that $\left|\left\langle\psi_{i} \mid \psi_{j}\right\rangle\right|^{2}=\frac{1}{d+1}$ whenever $i \neq j$. For now, whether SICs exist for all finite values of $d$ is an open research question. ${ }^{9}$ But, provided the relevant SICs exist, we find that an arbitrary quantum state $\rho$ can be expressed in terms of a fiducial SIC measurement $\Pi_{i}$ and the probability $p(i)$ of a subsequent measurement result:

$$
\rho=\sum_{i=1}\left((d+1) p(i)-\frac{1}{d}\right) \Pi_{i} .
$$

\footnotetext{
${ }^{9}$ So far SICs have been found for dimensions up to 151 , with several sparse analytic proofs up to 323 . See Fuchs et al. (2017) and the references therein.
} 
Consider a standard von Neumann measurement with outcomes $D_{j}=|j\rangle\langle j|$. Call the probability for this measurement given by the standard Born rule (2) $q(j)$. Now, suppose we perform a SIC measurement with outcomes $H_{i}$ before the von Neumann measurement. In this case, we can apply the law of total probability to arrive at the following:

$$
s(j)=\sum_{i=1}^{d^{2}} p(i) r(j \mid i)
$$

where $p(i)$ is the probability of an outcome $i$ of the SIC measurement, $r(j \mid i)$ is the conditional probability of an outcome $j$ of a subsequent von Neumann measurement conditional on $i$, and $d$ is the Hilbert space dimension associated with the system. But, this probability $s(j)$ differs from the Born rule probability $q(j)$ that applies if the SIC is not performed. In particular,

$$
q(j)=(d+1) s(j)-1 .
$$

Putting these expressions together, we arrive at the Essential Representation of the Born rule:

$$
q(j)=(d+1) \sum_{i=1}^{d^{2}} p(i) r(j \mid i)-1
$$

The QBist maintains that the Essential Representation (6) provides a more perspicuous version of the Born rule. Whereas the standard version of the Born rule relates a quantum state to the probability of a measurement outcome, the Essential Representation expresses a relation between probabilities associated with different sequences of measurements. According to the QBist, such probabilities should be understood as subjective degrees of belief, and hence, the Essential Representation functions as a kind of coherence constraint analogous to the axioms of (classical) probability theory. ${ }^{10}$ Moreover, this version of the Born rule allegedly "correlates with something that one might want to call 'real' " (Fuchs 2017b, p.6), and hence, we may wish to regard QBism as at least committed to this minimal claim about external reality. ${ }^{11} \mathrm{It}$ is also worth noting that Fuchs claims the dimension $d$ of the Hilbert space associated with a physical system — which appears in the Essential Representation-also indicates an objective feature of reality. In particular, it reflects a "previously unnoticed capacity inherent in all matter" (Fuchs 2010, p.23). ${ }^{12}$

\footnotetext{
${ }^{10} \mathrm{I}$ will return to this point in Section 5.1 below.

${ }^{11}$ What is intended by Fuchs here is far from clear. One might try to explicate this idea as a form of structural realism: the Born rule represents an aspect of the structure of our world. After all, structural realists frequently associate structure with what is encoded in the equations of physical theories. E.g.: "On the structural realist view what Newton really discovered are the relationships between phenomena expressed in the mathematical equations of his theory" (Worrall 1989, p.122).

${ }^{12}$ See Section 4.1 .
} 
Selective scientific realism allows for a range of positions depending on what aspects of models are taken to reflect genuine elements of reality. The present suggestion is that we locate QBism toward one end of the spectrum-in which almost nothing in the model corresponds to an element of reality. One may wonder whether such a view may be appropriately regarded as consistent with scientific realism. For some, scientific realism is equated with a belief in all of the unobservable entities posited by our best scientific theories. Even if one loosens this requirement somewhat, one may reasonably wonder whether a view that rejects so much of the apparent ontological commitments of quantum theory retains any significant portion of this motivating idea.

Another motivating idea for realists is the "no miracles" argument, which contends that (approximate) truth is required to explain the empirical success of our scientific theories. Moving away from the linguistic notion of truth as successful reference, we may capture this idea as the intuition that a model must reflect something in the world for its successful application to be unsurprising. Again, we may wonder whether merely latching onto one (albeit fairly central) feature of the world-the Born ruleis sufficient to explain the astonishing empirical success of quantum theory. Given that the Born rule - especially in the form of the Essential Representation-is purely relational, it's hard to see how it could meet this requirement. In order to generate the probability for a specific measurement outcome, one must supplement the Born rule with a quantum state or the probability of the outcome of another (hypothetical) measurement. But, given that these inputs to the Born rule are purely subjective on QBism, there's no guarantee that the theory will be successful.

Even if we put these issues to one side, there are further worries with adopting this perspective on QBism. The main one is that the resulting view is deeply uninformative. On this understanding, QBism posits an external reality and claims that the Born rule is (somehow) true of it. If there is nothing more to say, this has the effect of leaving the metaphysics of our world largely unconstrained. This leads to a view in which reality is fundamentally "unspeakable" (Timpson 2008) or "ineffable" (Brown 2019). Thus, the metaphysician is free to speculate on what the world is like, which could lead to radically different ontological pictures. For example, one might arrive at quasi-idealism ${ }^{13}$ (Brown 2019), Cartwrightian dispositionalism (Timpson 2008), Kantianism (Mohrhoff 2014) or countless other views.

Thus, the standard picture of scientific realism is an unhappy fit with QBism. If QBism is a genuine form a scientific realism, it cannot be in virtue of quantum models reflecting features of the world. The vague appeal to the real correlates of the Born rule (and Hilbert space dimension) is too thin a basis to support the realist's demands for explanation and understanding. One possible conclusion is that QBism

\footnotetext{
${ }^{13}$ Brown recognizes that "QBism is not strictly idealism in Berkeley's sense; it does not 'deny the existence of an observer-independent reality'," but he goes on to say, "...I find the ineffable nature of the external world in QBism troubling, and it is this concern that leads me to make the analogy with George Berkeley's metaphysics" (Brown 2019, p.19). The present point is that the very minimal metaphysical commitments of QBism are compatible with a variety of different views, and even if full-bodied idealism isn't among them, Brown suggests a kind of "quasi-idealism” may be.
} 
simply isn't a realist view, despite the urging of its proponents. ${ }^{14}$ But, there is some reason to resist this implication and instead challenge the vision of scientific realism on offer. After all, QBism rejects the idea that an interpretation of quantum theory is exhausted by what it tells us about external reality. It is unsurprising, then, that it is vague and uninformative when cast in these terms. For the QBist, while it may be true that we can glean some modest claims about such a reality from quantum theory, these are downstream consequences of the interpretation rather than its essence. This explains why the ineffable world isn't a problem for the QBist; understanding what the theory is telling us is divorced from the project of giving a metaphysics.

\section{Perspectivalism}

QBists emphasize the first-personal nature of quantum theory as one of the central tenets of QBism. In distinguishing his view from the Everettian interpretation of Wallace (2012), Fuchs notes that “...this is one of the most distinguished differences between Everett and QBism. 'QBism don't do third-person!' For QBism, all of quantum theory is first-person for the person who happens to be using it" (Fuchs 2017a, p.22). A common feature of standard scientific realism is that some features of models should correspond to the world in a perspective-independent sense. This implies that quantum models provide something like a God's eye description of the world. QBism rejects this framework by insisting that quantum theory only applies at the level of a particular agent embedded in the world in a particular way.

QBism isn't entirely unique in this respect. For example, Dieks's perspectivalism (Dieks 2009,2019) and Rovelli's relational interpretation (Rovelli 1996, 1997) also emphasize the role of agent perspectives in quantum theory. Both views reject the assumption of a single, God's eye quantum state for a given system at a time, but rather, insist on a plurality of observer-relative quantum states. Despite their essential appeal to observer perspectives, however, these interpretations still aim to provide a description of external reality on the basis of quantum theory. In light of this, they may be seen as compatible with standard scientific realism.

QBism goes further than these views by rejecting the goal of providing a description of external reality based in quantum theory. For Rovelli and Dieks, quantum states (and the properties they encode) are defined relative to agent perspectives. This suggests a metaphysics in which relational properties replace intrinsic (nonrelational) properties for physical systems. For instance, an electron may be $x$-spin "up" relative to Alice, but lack a determinate $x$-spin relative to Bob. For the QBist, by contrast, quantum theory is simply not in the business of giving this sort of a description of reality. Thus, the variety of perspectivalism implicated in QBism must

\footnotetext{
${ }^{14}$ Indeed, this is the conclusion drawn by (Hagar 2003) and (Norsen 2016). Given how little QBism has to say about reality, Hagar views it as ignoring the measurement problem thus as "instrumentalism in disguise" (Hagar 2003, p.772). Norsen goes further, labelling QBism "FAPP [for all practical purposes] solipsism" because it leaves the nature of reality external to the agent largly unconstrained (Norsen 2016, p.10). Even (Timpson 2008), who defends QBism's realist credentials from the charges of solipsism and instrumentalism, complains that the "unspeakable" nature of reality on QBism gives rise an explanatory deficit for the view.
} 
be stronger than merely asserting that quantum states or properties are defined relative to agent perspectives. The perspectivalism of QBism holds that quantum theory only applies at the level of agents embedded or situated in the world.

Following Ismael (2007), we can distinguish between conservative and radical varieties of perspectivalism. According to conservative perspectivalism about some discourse, a complete specification of the perspective-free facts together with the situation of the agent fixes the perspectival facts. For example, claims about what is nearby are fixed by distance relations and the location of the agent. Radical perspectivalism occurs when this fails- the perspectival facts don't supervene on the God's eye description together with a specification of the agent's situation. QBism should be viewed as radical perspectivalism about quantum theory in contrast to the conservative perspectivalism of Rovelli and Dieks. Even if we specify the details of an agent's situation and the God's eye facts about reality, this doesn't fix the quantum model that agent will adopt. Agents in the very same situation may assign different quantum states to the same system. ${ }^{15}$

\subsection{The participatory universe}

The perspectival nature of QBism helps to make sense of another distinctive feature of QBism. Inspired by John Wheeler, QBists have sought to emphasize the "participatory" aspect of quantum theory.

When an experimentalist reaches out and touches a quantum system-the process usually called quantum 'measurement' - that process gives rise to a birth. It gives rise to a little act of creation. And it is how those births or acts of creation impact the agent's expectations for other such births that is the subject matter of quantum theory. (Fuchs 2017b, p.9)

This talk of measurements as acts of creation suggests a metaphysical picture in which we construct the world via our interactions with it. This may be further supported by consideration of the role played by Hilbert space dimension, which figures in the Essential Representation and which Fuchs (2010) describes as a "universal capacity" of quantum systems. Indeed, Timpson (2008) suggests an ontology of primitive capacities on the basis of such considerations. Now, there is nothing obviously anti-realist about a metaphysics in which humans build the world bit by bit via their interactions with it-if quantum theory is telling us that this is how reality operates, then as realists we had better endorse it.

But, such a picture does raise a number of questions. If agents construct reality via their measurements, how do we make sense of different agents performing different

\footnotetext{
${ }^{15}$ It could be argued that perspectival facts (e.g., measurement outcomes) ultimately supervene on the God's eye facts and the agent's situation-it's just that the former are largely unknowable according to QBism. However, there remains an important difference between QBism and other varieties of perspectivalism: Only QBism posits perspectival facts that fail to supervene on the accessible perspective-free facts together with a suitable specification of the agent's situation. This reflects the greater autonomy QBism allows in how agents apply quantum theory to the world. Thanks to an anonymous referee for raising this issue.
} 
measurements with different results? Indeed, the case of Wigner's friend-usually taken to support epistemic approaches like QBism-would seem to lead to a paradoxical situation in which the world is more than one way, or in which Wigner and his friend occupy different worlds. Fortunately, these puzzles are avoided when we recall the perspectival aspect of QBism discussed above.

According to QBism, the primary function of quantum theory is to guide agents in their beliefs, not to describe external reality. This means it is a mistake to elevate what it says about an individual agent's experiences to the level of a God's eye description of the world. Indeed, a central tenet of the view is that quantum theory can provide no such God's eye description. Thus, while it may be true from the perspective of an individual agent that she creates the world via her measurements, this should not be taken as a metaphysical proposal about external reality.

Now, one might object that this account doesn't do justice to the participatory aspect of QBism. It may seem that the language of "acts of creation" is being dismissed as mere metaphor rather than a genuine aspect of the quantum world. But, we must recall that on QBism there is no such thing as the quantum world, at least if that's taken to mean the world described by quantum theory. Rather, quantum theory functions as a guide to agents. The participatory universe and the claim that measurements are acts of creation, then, should be understood at the level of description of an agent applying quantum theory to their world. Whether this is true will depend on whether measurements really are acts of creation, when viewed from the perspective of an agent applying quantum theory. And this seems plausible. For an agent embedded in a world like ours, quantum theory instructs them to expect a certain measurement outcome according to the Born rule probability assigned to it and not to view such measurements as revealing a preexisting physical property. If this were not the case - say, if there were no superposition principle in quantum theory-then, it would be false (or at least we would be unjustified in believing) that measurements are acts of creation. So, it is plausible that features of our world and features about us as agents combine to make it the case that, from the perspective of an agent using quantum theory, measurements are indeed acts of creation.

As an analogy, consider the case of color, understood as a secondary quality. On a simple version of this view, to be red is to appear red to a person under ordinary conditions. In one sense, objects are not colored on this view-objects are not red from a God's eye perspective. However, it is not as though someone describing a tomato as red is making a mistake or falling victim to an illusion in light of this fact. Rather, "being red" is a property that makes essential reference to a potential observer. The present proposal is to treat measurement outcomes (and other aspects of quantum models) similarly. They are not features of external reality, but features of agents. These perspectival facts may ultimately be grounded in facts about external reality, but the connection is not straightforward. ${ }^{16}$

\footnotetext{
${ }^{16}$ Cf., Evans (2020) who uses the example of color to motivate what he calls "perspectival objectivity," which refers to "a scenario in which some feature of the world is in part a function of the agent perspective while at the same time, given such a perspective that is inescapably shared between similar agents, there is an (intersubjectively) objective fact of the matter concerning that feature" (2020, p.19).
} 
Thus, the QBist can say that agents create their own worlds by performing measurements without being compelled to deny that agents inhabit a single reality. The former should be viewed as a non-metaphysical claim to be assessed in the context of the situated perspective while the latter should be viewed as a metaphysical claim about objective reality. Now, it may be thought that QBism's rejection of the God's eye perspective renders QBists unable to say anything about objective reality, but this cannot be right. For as we have seen, QBists wish to claim that there is an external reality independent of our thoughts of it (this is what kicks back when we prod it) and that we can know certain things about it (e.g., that it supports our use of the Born rule). The denial of a God's eye description is a claim about the limits of quantum theory, not the impossibility of metaphysics by other means.

\section{Normativity}

There is a sense in which any interpretation of quantum theory has normative implications, namely, that one should expect to find what the formalism predicts. However, on many interpretations, such normative prescriptions follow from the truth of the description of reality the theory is taken to provide. By contrast, on QBism, quantum normative claims do not follow from a description of reality in any straightforward sense. Rather, quantum theory is taken to be fundamentally normative, not descriptive. This is a point QBism shares with other non-ontic approaches, such as Healey's pragmatism. An important difference between the two views is in what they take the normative content, or prescriptions, of quantum theory to be. For Healey, an agent's quantum state ascriptions taken individually are subject to normative constraints; there is an objective fact about which quantum state an agent should ascribe in a given physical context. QBists, however, only recognize a relational normative constraint in the Born rule (in the form of the Essential Representation). As Fuchs (2017a) says, "[n]othing is sacred except that [an agent] should strive to satisfy the Born Rule for all probabilities" (p.13). What both views share is that quantum theory imposes objective normative constraints on all agents (see Healey 2017a).

If we take this seriously, it has important implications for the questions of realism about quantum theory. In particular, if it is a normative theory, then realism about quantum theory is a form of normative realism. And while there is much controversy surrounding normative realism, at least some prominent philosophers have advocated a position analogous to QBism's take on quantum theory. Consider, for instance, how T.M. Scanlon characterizes his version of normative realism:

...the point of judgments of right and wrong is not to make claims about what the spatiotemporal world is like. The point of such judgments is, rather, a practical one: they make claims about what we have reason to do. Metaphysical questions about the subject matter of judgments of right and wrong are important only if answers to them are required in order to show how these judgments can have this practical significance. (Scanlon 1998, p.2)

For Scanlon, moral metaphysics is only relevant to the extent that it is needed to account for the prescriptions of morality. For QBists, who view quantum theory 
normatively, the parallel point is that quantum metaphysics is only relevant to the extent that one needs it to account for the prescriptions of quantum theory-i.e., the constraints imposed by the Born rule. As long as one can provide such an account, there are some grounds for regarding the view as a form of realism. After all, it maintains that the dictates of quantum theory are objective and is able to say why they are important. ${ }^{17}$

\subsection{Grounding normativity}

QBism maintains that the significance of the Born rule-formulated purely in terms of subjective probabilities-is to tell us what we have reason to do. If this role can be sustained without getting involved in metaphysical questions, then QBism can maintain some measure of objectivity without making substantial claims about external reality. However, there is an obvious problem with such a claim in the context of quantum theory (whether it is successful in ethics is another question): How, without providing a description of the world, can we say that we have reason to do what the Born rule prescribes? Without an answer to this question, the scientific realist will be unsatisfied as the central (normative) content of the theory is left unaccounted for.

One strategy for grounding the normative prescriptions issued by quantum theory is inductive in nature. It says that we should do as quantum theory recommends because (1) this is what it is to accept quantum theory and (2) we should accept quantum theory on the basis of its past success. This is the approach of Healey (2017b).

To accept quantum theory is to commit oneself to setting credence in each significant canonical magnitude claim equal to the probability specified by a legitimate application of the Born rule based on the best available quantum model, in the absence of more direct access to the truth value of the claim. (Healey 2017b, p.131)

He goes on to defend point (2):

The strongest reason to accept quantum theory is provided by the success of its applications in predicting and explaining physical phenomena of a statistical nature. If we set credences in accordance with the Born rule we are led to expect and can come to understand the patterns displayed by these statistics. (Healey 2017b, p.131)

However, it's not clear that this strategy is available to the QBist. First, measurement outcomes are not objective features of reality according to QBism, so there are no objective frequencies to appeal to. Each agent will have their own memories of measurement experiences, which are the result of their interactions with the world,

\footnotetext{
${ }^{17}$ Whether one regards such a normative realism as scientific realism is another matter. I will simply assume that if one is a realist with respect to a scientific theory, then one is a (local) scientific realist. In the present case, if quantum theory is a normative theory as QBists claim, then normative realism about quantum theory is sufficient for scientific realism about quantum theory.
} 
but will differ between agents. Second, the Born rule alone doesn't make any predictions. One needs to supplement the Born rule with a quantum state ascription-or the probability of another measurement outcome in the Essential Representationto arrive at a specific probability. This relational nature of the Born rule means that measurement statistics cannot bear on it directly. This isn't a problem for Healey, as he maintains that quantum state ascription is objective (though relational), but QBists demur. ${ }^{18}$ So, it seems QBism lacks the resources to offer an inductive grounding of the Born rule. ${ }^{19}$

Instead, QBists adopt a strategy for grounding normativity that borrows from the subjectivist tradition in probability. Bruno de Finetti famously denied that there is anything in external reality corresponding to our judgments of probabilities, and hence, was compelled to offer an alternative account of the axioms of probability theory. This alternative was the Dutch book approach, which views the probability axioms as coherence constraints. In terms of betting behavior, if one doesn't adhere to the axioms, they will be vulnerable to a "Dutch book": a series of fair bets in which they are guaranteed to lose money regardless of which outcomes obtain (Ramsey 1926; De Finetti 1937). Of course, there needn't be any actual "Dutch bookies" lurking. The point is rather that the possibility of such sequences of bets reveals an internal inconsistency in one's degrees of belief.

Fuchs and Schack (2013) argue that the Essential Representation of the Born rule should be viewed in along the same lines:

It expresses a kind of 'empirically extended coherence' not implied by Dutchbook coherence alone, but formally similar to the kind of relation one gets from Dutch-book coherence. (Fuchs and Schack 2013, p.1702)

The proposal is to afford the Born rule a similar status to the axioms of probability theory taken subjectively; if we don't follow the Born rule in our subjective degrees of belief, we are being incoherent. In terms of betting behavior, violating the Born rule opens one up to the possibility of a Dutch book that guarantees a sure loss. There

\footnotetext{
${ }^{18}$ Presumably, other interpretations would also be able to appeal to quantum theory's track record. The difference is that the standard realist interpretations also appeal to the fact that the world is as quantum theory describes it to be. Healey's view, by contrast, maintains only that quantum theory's past success grounds our commitment to its prescriptions. Thanks to an anonymous referee for raising this point.

${ }^{19}$ While QBism is able to accommodate the measurement results that form the inductive evidence for quantum theory, it cannot ground our use of the Born rule in these data. Measurement outcomes are elements of subjective experience on QBism, and as such are completely unconstrained. Now, some agents may happen to have past experiences that conform to the Born rule, but there is no guarantee of this. Moreover, the Born rule requires a particular quantum state assignment (or, in the case of the Essential Representation, the probability of an additional hypothetical measurement) to generate a specific probability. So, the most one can appeal to here are subjective measurement records in conformity with the Born rule when supplemented with certain subjective quantum state assignments (or subjective measurement probabilities). This fails to provide an inductive grounding that is objective in the sense of providing a compelling reason for all agents to use quantum theory. Thanks to an anonymous referee for raising this worry.
} 
is a crucial difference between the cases, however: the quantum Dutch book, unlike the standard Dutch book, requires more than logic and mathematics. The quantum Dutch book applies only in a world relevantly similar to ours-one in which quantum theory provides a good guide for agents in it.

But what is it about our world that makes the Born rule the objectively correct coherence constraint? QBists have two sorts of replies to this question. First, they may leave this as a brute feature of reality. That the Born rule acts as a coherence constraint is the limit of what we can say about the world. As Fuchs says, it is "nature's whisper" (Fuchs 2017b, p.6). Sometimes QBists express a desire to say more, but note that QBism is an active research project, and as such, does not have all of the answers at present. So, a second approach is to seek out the features of our world that necessitate the use of the Born rule. One way to do this would be to derive the Born rule from logic and mathematics supplemented with a minimal empirical claim. However, it's hard to see what resources the QBist has at their disposal for this task. For instance, consider the approach of a non-QBist, Pitowsky (2003), who argues that quantum probability (i.e., the Born rule) follows from rational betting on quantum gambles. The empirical premise in Pitowsky's derivation is the assumption that the algebra formed by the outcomes of incompatible quantum measurements has a non-Boolean structure. ${ }^{20}$ But, such a claim is incompatible with QBism's subjective understanding of measurement outcomes, which places no constraints on what an individual may experience. So, while such a derivation may eventually be possible, it's presently unclear what empirical premises would be involved. At present, then, QBism must rest content with the first approach: it is a brute fact that the Born rule acts as a coherence constraint.

This picture may be unsatisfying for traditional scientific realists, but recall that according to QBism quantum theory is, first and foremost, a normative theory. Hence, the version of realism according to which it should be assessed is one appropriate for normative theories. If we follow the approach suggested by Scanlon, such a realism must account for the reason-giving force of the prescriptions of the theory in question - in this case, those following from the Born rule. The Dutch book approach does this. Because it is an objective coherence constraint for agents in a world like ours, the Born rule gives us reason to set our credences in conformity with it. Now, one may wish to know why the Born rule acts as a coherence constraint in our world and, for the moment, there is very little the QBist can say here. But, again, this is

\footnotetext{
${ }^{20}$ More specifically, a quantum gamble proceeds in four stages:
}

1. A single physical system is prepared by the bookie.

2. A finite set $\mathcal{M}$ of incompatible measurements is announced by the bookie, and the agent is asked to place bets on possible outcomes of each one of them.

3. One of the measurements in the set $\mathcal{M}$ is chosen by the bookie and the money placed on all other measurements is promptly returned to the agent.

4. The chosen measurement is performed and the agent gains or loses in accordance with his bet on that measurement.

The empirical assumption is that the quantum gambler is aware of the Boolean algebras corresponding to the outcomes of two incompatible measurements. When considered together, these outcomes form a non-Boolean algebra (Pitowsky 2003, pp. 396-397). 
unsurprising given that QBism rejects the idea that quantum theory functions as a description of reality.

In sum, QBism should be understood as a perspectival normative realism according to which quantum theory is prescriptive rather than descriptive, and perspectival rather than applied from the God's eye view. Whether such a position counts as realism depends on the existence of grounds for the normative claims involved in quantum theory, so understood. The inductive approach is unavailable to the QBist, but the Dutch book approach provides another option. For now, QBism is unable to identify the specific features of our world that ground its use, but the Dutch book approach may nevertheless account for why we should set our credences in accordance with the Born rule.

\section{Conclusion: meeting realist demands}

The aim of this paper has been to investigate the relation of QBism to scientific realism. The conclusion we have reached is partially negative: standard ways of thinking about scientific realism are an unhappy fit with QBism. While QBism may perhaps be viewed as a limiting case of selective scientific realism-one in which almost no aspects of quantum theory correspond to elements of reality, the resultant picture is highly uninformative. Standard scientific realism also fails to take seriously the perspectival and normative aspects of QBism, according to which quantum theory does not offer a God's eye description of reality, but places objective normative constraints on agents. The question remains, whether this perspectival normative realism suffices for scientific realism.

There are several criteria that could be used to demarcate scientific realisms from antirealisms. If these include a commitment to providing a substantive description of reality from the God's eye view, QBism straightforwardly fails. But, such accounts may be seen as question-begging given QBism's rejection of this conception of what quantum theory aims to do. But other criteria seem more central to scientific realism. For instance, realism is often associated with the ability to provide explanations of certain phenomena. Some have objected to QBism on precisely these grounds:

...QBism seems explanatorily inert. For scientific explanations typically explain phenomena in terms of underlying mechanisms. Here is a simple example. Why is the Sun able to produce so much energy over such a long period of time? (McQueen 2017, p.7)

McQueen (2017) goes on to argue that QBism can provide no underlying physical mechanism responsible for the sun's energy production (presumably because our understanding of the nuclear processes involved depends on quantum models which fail to describe reality according to QBism). But, it's question-begging to demand of QBism that it provides causal-mechanistic explanations of quantum phenomena, given that one of its central features is a rejection of quantum theory's alleged descriptive role. Moreover, QBism can offer a certain kind of explanation of phenomena in the scope of quantum theory: the QBist can note that quantum theory tells agents 
that they should expect to find certain things. ${ }^{21}$ McQueen considers such an explanation of his case, but quickly rejects it for failing to account for why we should expect these phenomena to occur (ibid.). And so we are back to the question of grounding the objective normative prescriptions of quantum theory. McQueen assumes that a description of reality based in quantum theory is needed to fully account for the phenomena about which it advises agents, but as we have seen, there are alternative ways to ground normativity.

The adequacy of the explanations licensed by QBism is an open question, and an issue that may motivate some to reject QBism. The central point here is that to dismiss QBism's claim to realism on the basis of a lack of causal-mechanistic explanations is to assume the standard conception of scientific realism. Above, I have argued that this is not the appropriate conception of realism for a view that takes quantum theory to be radically perspectival and fundamentally normative.

By way of conclusion, consider again Scanlon's moral realism. ${ }^{22}$ Shortly after the quotation above, Scanlon outlines two worries for realism about ethics without an underlying moral metaphysics:

One worry would be that there may be no right answer to questions of right and wrong... A second interpretation of the charge that judgments of right and wrong are not about anything "real" would take it as the claim that they should not have this importance. This is a charge that any account of the reason-giving force of judgments of right and wrong needs to meet. (Scanlon 1998, pp.2-3)

Applied to QBism, the first worry is that the QBist cannot maintain that there are objectively correct answers to questions about how to set our credences. The second worry is that only an adequate description of the world can account for the reasongiving force of quantum theory and the Born rule. QBism meets the first challenge by regarding the Born rule - in the form of the Essential Representation and together with the probability axioms - as an objective coherence constraint. While there are not correct answers to individual probability judgments, there are constrains on the set of admissible credence functions, namely, that they must conform to the Born rule. The second challenge is met by the Dutch book approach. The prescriptions of the Born rule have importance because all rational agents are compelled to adhere to

\footnotetext{
${ }^{21}$ Recall that the normative prescriptions of quantum theory are solely relational according to QBism, so explanations will take the form of prescribed relations between beliefs. Nevertheless, in many cases, quantum theory is capable of telling agents that they should expect to attain certain results in light of their other beliefs.

${ }^{22}$ I've only considered a small part of Scanlon's views here. One might wonder whether other aspects of his views may be usefully applied to QBism. For instance, according to his contractualism, ethical principles are justified by the fact that no reasonable person can deny their truth. Could QBists take a similar attitude toward quantum theory? Is the Born rule a normative constraint that no reasonable person can deny? Possibly, but if so its undeniability is parasitic on a grounding of the sort considered above. Absent such a grounding, there is little reason to suppose that the Born rule is rationally undeniable. Indeed, the very reason we seek such a grounding is to account for the apparent reason-giving force of quantum theory. Thus, while other aspects of Scanlon's view may be helpful in further developing QBism, the key idea for present purposes is simply that realism about a normative theory needn't require an underlying metaphysics. Thanks to an anonymous referee for this suggestion.
} 
them, in the same manner as the axioms of subjective probability theory. Unlike the probability axioms, there is no a priori proof for quantum theoretic constraints, but QBists maintain that they capture an empirically extended sense of rational coherence. According to QBism, quantum theory provides correct answers to questions about what we should do, and provides us with reasons to do as it prescribes. Thus, it would seem that QBism can meet the demands of a conception of realism appropriate for a normative theory.

Open Access This article is licensed under a Creative Commons Attribution 4.0 International License, which permits use, sharing, adaptation, distribution and reproduction in any medium or format, as long as you give appropriate credit to the original author(s) and the source, provide a link to the Creative Commons licence, and indicate if changes were made. The images or other third party material in this article are included in the article's Creative Commons licence, unless indicated otherwise in a credit line to the material. If material is not included in the article's Creative Commons licence and your intended use is not permitted by statutory regulation or exceeds the permitted use, you will need to obtain permission directly from the copyright holder. To view a copy of this licence, visit http://creativecommons.org/licenses/by/4.0/.

\section{References}

Bacciagaluppi, G. (2014). A critic looks at QBism. In Galavotti, M.C., Dieks, D., Gonzalez, W.J., Hartmann, S., Uebel, T., Weber, M. (Eds.) New directions in the philosophy of science (pp. 403-416). Cham: Springer.

Boge, F.J. (2018). Quantum mechanics between ontology and epistemology volume 10 of European studies in philosophy of science. Cham: Springer.

Brown, H.R. (2019). The reality of the wavefunction: Old arguments and new. In Cordero, A. (Ed.) Philosophers look at quantum mechanics (pp. 63-86). Cham: Springer International Publishing.

Caves, C.M., Fuchs, C.A., Schack, R. (2002). Quantum probabilities as Bayesian probabilities. Physical Review A, 65(2), 022305.

Caves, C.M., Fuchs, C.A., Schack, R. (2007). Subjective probability and quantum certainty. Studies in History and Philosophy of Science Part B: Studies in History and Philosophy of Modern Physics, 38(2), 255-274.

De Finetti, B. (1937). Foresight: Its logical laws, its subjective sources. In Henry, E., \& Kyburg, H.E.S. (Eds.) Studies in subjective probability. Huntington: Robert E. Kreiger Publishing Co.

DeBrota, J.B., Fuchs, C.A., Stacey, B.C. (2020). Symmetric informationally complete measurements identify the irreducible difference between classical and quantum systems. Physical Review Research, 2(1), 013074.

Dieks, D. (2009). Objectivity in perspective: relationism in the interpretation of quantum mechanics. Foundations of physics, 39(7), 760-775.

Dieks, D. (2019). Quantum mechanics and perspectivalism. arXiv:1801.09307v2.

Earman, J. (2019). Quantum Bayesianism assessed. The Monist, 102(4), 403-423.

Evans, P.W. (2020). Perspectival objectivity. European Journal for Philosophy of Science, 10(2), 19.

Friederich, S. (2013). In defence of non-ontic accounts of quantum states. Studies in History and Philosophy of Science Part B: Studies in History and Philosophy of Modern Physics, 44(2), 77-92.

Friederich, S. (2015). Interpreting quantum theory: a therapeutic approach. Basingstoke: Palgrave Macmillan.

Fuchs, C.A. (2010). QBism, the perimeter of quantum Bayesianism. arXiv:1003.5209.

Fuchs, C.A. (2017a). Notwithstanding Bohr, the reasons for QBism. Mind and Matter, 15(2), 245-300.

Fuchs, C.A. (2017b). On participatory realism. In Durham, I.T., \& Rickles, D. (Eds.) Information and interaction: Eddington, wheeler, and the limits of knowledge (pp. 113-134). Cham: Springer International Publishing.

Fuchs, C.A., Hoang, M.C., Stacey, B.C. (2017). The SIC question: History and state of play. Axioms, 6(3), 21.

Fuchs, C.A., \& Schack, R. (2013). Quantum-Bayesian coherence. Reviews of Modern Physics, 85, 16931715 . 
Hacking, I. (1983). Representing and intervening: Introductory topics in the philosophy of natural science. Cambridge: Cambridge University Press.

Hagar, A. (2003). A philosopher looks at quantum information theory. Philosophy of Science, 70(4), 752775.

Harrigan, N., \& Spekkens, R.W. (2010). Einstein, incompleteness, and the epistemic view of quantum states. Foundations of Physics, 40(2), 125-157.

Healey, R. (2012). Quantum theory: a pragmatist approach. The British Journal for the Philosophy of Science, 63(4), 729-771.

Healey, R. (2017a). Quantum-Bayesian and pragmatist views of quantum theory. In Zalta, E.N. (Ed.) The stanford encyclopedia of philosophy. Metaphysics Research Lab, Stanford University, spring 2017 edition.

Healey, R. (2017b). The quantum revolution in philosophy. Oxford: Oxford University Press.

Healey, R. (2020). Pragmatist quantum realism. In Saatsi, J., \& French, S. (Eds.) Scientific realism and the quantum, chapter 7 (pp. 123-146). New York: Oxford University Press.

Ismael, J. (2007). Causation, perspective, and agency. Available online at http://www.u.arizona.edu/ jtismael/6.Causation, pers., agency.pdf.

Laudan, L. (1981). A confutation of convergent realism. Philosophy of Science, 48(1), 19-49.

McQueen, K.J. (2017). Is QBism the future of quantum physics? arXiv:1707.02030.

Mermin, N.D. (2014). QBism in the new scientist. arXiv:1406.1573.

Mohrhoff, U. (2014). QBism: a critical appraisal. arXiv:1409.3312.

Myrvold, W. (2018). Philosophical issues in quantum theory. In Zalta, E.N. (Ed.) The stanford encyclopedia of philosophy. Metaphysics Research Lab, Stanford University, fall 2018 edition.

Norsen, T. (2016). Quantum solipsism and non-locality. In Bell, M., \& Gao, S. (Eds.) Quantum nonlocality and reality: 50 years of Bell's theorem (pp. 204-237): Cambridge University Press.

Pitowsky, I. (2003). Betting on the outcomes of measurements: a Bayesian theory of quantum probability. Studies in History and Philosophy of Science Part B: Studies in History and Philosophy of Modern Physics, 34(3), 395-414.

Psillos, S. (1999). Scientific realism: How science tracks truth. New York: Routledge.

Pusey, M.F., Barrett, J., Rudolph, T. (2012). On the reality of the quantum state. Nature Physics, 8(6), 475.

Ramsey, F.P. (1926). Truth and probability. In Henry, E., \& Kyburg, H.E.S. (Eds.) Studies in Subjective Probability. Huntington: Robert E. Kreiger Publishing Co.

Rovelli, C. (1996). Relational quantum mechanics. International Journal of Theoretical Physics, 35(8), $1637-1678$.

Rovelli, C. (1997). Half way through the woods. In Earman, J., \& Norton, J. (Eds.) The Cosmos of Science (pp. 180-223). Pittsburg: University of Pittsburg Press.

Scanlon, T. (1998). What we owe to each other. Cambridge: Harvard University Press.

Spekkens, R.W. (2007). Evidence for the epistemic view of quantum states: a toy theory. Physical Review A, 75(3), 032110 .

Timpson, C.G. (2008). Quantum Bayesianism: A study. Studies in History and Philosophy of Science Part B: Studies in History and Philosophy of Modern Physics, 39(3), 579-609.

van Fraassen, B. (1980). The scientific image. Oxford: Oxford University Press.

Wallace, D. (2012). The emergent multiverse: Quantum theory according to the Everett interpretation. Oxford: Oxford University Press.

Wolff, J. (2020). Naturalism and the interpretation of quantum mechanics. In French, S., \& Saatsi, J. (Eds.) Scientific realism and the quantum (pp. 103-120). Oxford: Oxford University Press.

Worrall, J. (1989). Structural realism: The best of both worlds? Dialectica, 43(1-2), 99-124.

Publisher's note Springer Nature remains neutral with regard to jurisdictional claims in published maps and institutional affiliations. 